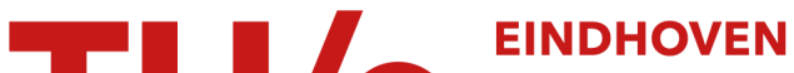 \\ UNIVERSITY OF \\ TECHNOLOGY
}

\section{Surface induced asymmetry of acceptor wave functions}

\section{Citation for published version (APA):}

Celebi, C., Garleff, J. K., Silov, A. Y., Yakunin, A. M., Koenraad, P. M., Roy, van, W., Tang, J-M., \& Flatté, M. E. (2010). Surface induced asymmetry of acceptor wave functions. Physical Review Letters, 104(8), 086404-1/4. [086404]. https://doi.org/10.1103/PhysRevLett.104.086404

DOI:

10.1103/PhysRevLett.104.086404

Document status and date:

Published: 01/01/2010

\section{Document Version:}

Publisher's PDF, also known as Version of Record (includes final page, issue and volume numbers)

\section{Please check the document version of this publication:}

- A submitted manuscript is the version of the article upon submission and before peer-review. There can be important differences between the submitted version and the official published version of record. People interested in the research are advised to contact the author for the final version of the publication, or visit the $\mathrm{DOI}$ to the publisher's website.

- The final author version and the galley proof are versions of the publication after peer review.

- The final published version features the final layout of the paper including the volume, issue and page numbers.

Link to publication

\section{General rights}

Copyright and moral rights for the publications made accessible in the public portal are retained by the authors and/or other copyright owners and it is a condition of accessing publications that users recognise and abide by the legal requirements associated with these rights.

- Users may download and print one copy of any publication from the public portal for the purpose of private study or research.

- You may not further distribute the material or use it for any profit-making activity or commercial gain

- You may freely distribute the URL identifying the publication in the public portal.

If the publication is distributed under the terms of Article 25fa of the Dutch Copyright Act, indicated by the "Taverne" license above, please follow below link for the End User Agreement:

www.tue.nl/taverne

Take down policy

If you believe that this document breaches copyright please contact us at:

openaccess@tue.nl

providing details and we will investigate your claim. 


\title{
Surface Induced Asymmetry of Acceptor Wave Functions
}

\author{
C. Çelebi, J. K. Garleff, A. Yu. Silov, A. M. Yakunin, and P. M. Koenraad \\ COBRA Inter-University Research Institute, Department of Applied Physics, Eindhoven University of Technology, \\ P.O. Box 513, NL-5600 MB Eindhoven, The Netherlands \\ W. Van Roy \\ IMEC, Kapeldreef 75, B-3001 Leuven, Belgium \\ J.-M. Tang \\ Department of Physics, University of New Hampshire, Durham, New Hampshire 03824, USA
}

M. E. Flatté

Optical Science and Technology Center and Department of Physics and Astronomy, University of Iowa, Iowa City, Iowa 52242, USA (Received 28 January 2009; published 26 February 2010)

\begin{abstract}
Measurements of the local density of states of individual acceptors in III-V semiconductors show that the symmetry of the acceptor states strongly depends on the depth of the atom below a (110) surface. Tight-binding calculations performed for a uniformly strained bulk material demonstrate that strain induced by the surface relaxation is responsible for the observed depth-dependent symmetry breaking of acceptor wave functions. As this effect is strongest for weakly bound acceptors, it explains within a unified approach the commonly observed triangular shapes of shallow acceptors and the crosslike shapes of deeply bound acceptor states in III-V materials.
\end{abstract}

DOI: 10.1103/PhysRevLett.104.086404

In the past decade many groups have scrutinized at the atomic scale the electronic and spectroscopic details of individual impurities, such as $\mathrm{Si}, \mathrm{Zn}$, and $\mathrm{Mn}$, embedded in the topmost layers of a cleaved semiconductor crystal like GaAs or InAs [1-6]. A correct interpretation of these atomistic properties is essential for a proper understanding of the macroscopic properties of doped materials. For example, present models of impurity band formation and ferromagnetism [7] in GaMnAs require a proper description of the atomic scale properties of individual Mn acceptors. The surface, however, is known to strongly influence the properties of an impurity close to the surface [8], and thus a central question for all such surface measurements via cross-sectional scanning tunneling microscopy (XSTM) is how closely the local density of states (LDOS) is related to properties of an impurity in the bulk. Several groups have interpreted the XSTM contrast of acceptors as being directly related to the wave function of the impurity state $[2,3,5,6]$, while others have proposed that highly asymmetric observed acceptor shapes arise from hybridization of the impurity state with excited states [4] or intrinsic surface states [9].

Here we report measurements and calculations of the LDOS of individual Mn acceptors in GaAs as a function of their distance below the reconstructed surface, and show that the XSTM contrast can be fully understood from the wave function of the impurity state. The contrast changes from a highly asymmetric shape, for Mn acceptors in the topmost layers, to a symmetric shape, for Mn acceptors at 8 atomic layers (AL) below the surface. By comparison of measurements with theoretical calculations we identify
PACS numbers: 71.55.Eq, 68.37.Ef, 71.15.-m, 75.50.Pp

strain, induced by the surface reconstruction, as the dominant cause of the depth-dependent asymmetry of the LDOS for these energetically deep Mn acceptors in GaAs ( $E_{a}=$ $113 \mathrm{meV})$. We conclude that the LDOS of a Mn acceptor deep enough below the surface reflects the properties of the unperturbed acceptor state. For shallowly bound impurities like $\mathrm{Zn}$ in $\operatorname{GaAs}\left(E_{a}=31 \mathrm{meV}\right)$, with larger Bohr radii, this situation is never reached, and thus the observed LDOS of shallowly bound impurities is always strongly affected by the surface strain. Thus we resolve the longstanding controversy of XSTM contrast as due to the acceptor wave function, and simultaneously resolve the source of the very asymmetric LDOS of shallowly bound acceptors [10].

The XSTM experiments are performed on molecularbeam-epitaxy grown $\mathrm{Mn}$ doped $\mathrm{GaAs}$ and liquidencapsulated Czochralski grown $\mathrm{Zn}$ doped $\mathrm{GaP}$ and $\mathrm{Cd}$ doped GaP samples. The concentration of $\mathrm{Mn}$ in the GaAs epilayer was $2 \times 10^{18} \mathrm{~cm}^{-3}$. The bulk concentration of $\mathrm{Zn}$ in $\mathrm{GaP}$ and $\mathrm{Cd}$ in $\mathrm{GaP}$ was close to $5 \times 10^{17} \mathrm{~cm}^{-3}$. A clean and atomically flat surface was obtained by cleaving the samples inside the ultrahigh vacuum STM chamber with a base pressure $P<2 \times 10^{-11}$ torr. The topography scans were carried out on the (110) sample surface by using electrochemically etched polycrystalline tungsten tips that were further treated in vacuum as described in [11]. The measurement presented in Fig. 1 was acquired at $T=5 \mathrm{~K}$ in constant-current mode and the tunnel setpoint $V_{s}=+1.55 \mathrm{~V}, I_{t}=50 \mathrm{pA}$.

The topography image of Mn doped GaAs (Fig. 1) shows a number of $\mathrm{Mn}$ induced contrasts at the 


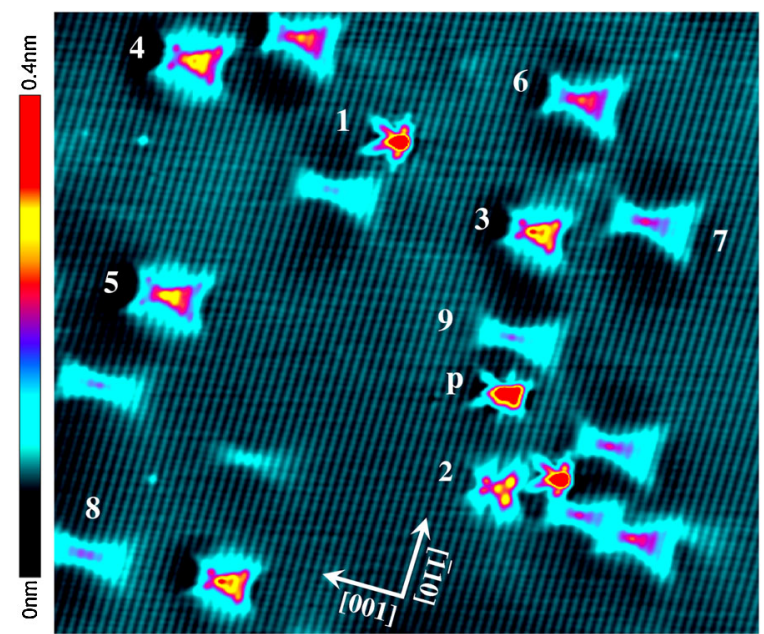

FIG. 1 (color online). (a) $\left(45 \times 35 \mathrm{~nm}^{2}\right)$ constant-current topography map of the GaAs(110) surface showing a number of $\mathrm{Mn}$ acceptors in their neutral charge state. The numbers correspond with the atomic layer position of the Mn acceptors in GaAs.

GaAs(110) cleavage surface. The numbers in the image correspond with the position of $\mathrm{Mn}$ acceptors below the GaAs(110) surface, where 1 indicates the surface layer. The depth determination and hence the labeling are done according to the relative intensity and symmetry of the $\mathrm{Mn}$ induced contrasts. The structure $p$ displays the typical shape of a Mn atom that is coupled to an adsorbate in the GaAs surface layer. The characteristics of the LDOS of these $\mathrm{Mn}$ related features were studied in detail elsewhere $[11,12]$. Similar to the Mn acceptors in InAs [5], we observe that Mn displays a contrast which changes from a trapezoidal to a crosslike pattern as a function of the depth of the $\mathrm{Mn}$ atom below the GaAs(110) surface. Unlike deeply buried $\mathrm{Mn}$, the contrast of a Mn acceptor close to the surface has a smaller extension and is more asymmetric in the GaAs(110) mirror plane. The contrast of the Mn acceptor located deeper than 8 AL below the surface (corresponding to the label 9 in Fig. 1) has a highly extended profile which is asymmetric only around the topography maximum. A similar profile of the Mn wave functions has been found in tight-binding (TB) calculations [13].

A pronounced quantitative depth dependence in the (001)-reflection asymmetry of the Mn acceptors can be seen in Fig. 2 where Fig. 2(a) depicts the measured height profiles of the Mn acceptors that are located between 2 and 8 AL below the surface. The profiles shown in Fig. 2(a) were obtained by averaging a number of parallel STM traces taken in the [001] direction across the center of the $\mathrm{Mn}$ acceptor contrast. The width of the box, in which the parallel [001] traces are averaged, is typically $0.5 \mathrm{~nm}$ in the [110] direction. To quantify the observed trends in the depth-dependent change of the Mn symmetry, the background atomic corrugation is filtered out from the topography line profiles by a fast Fourier transform (FFT) technique, and shown in Fig. 2(b).
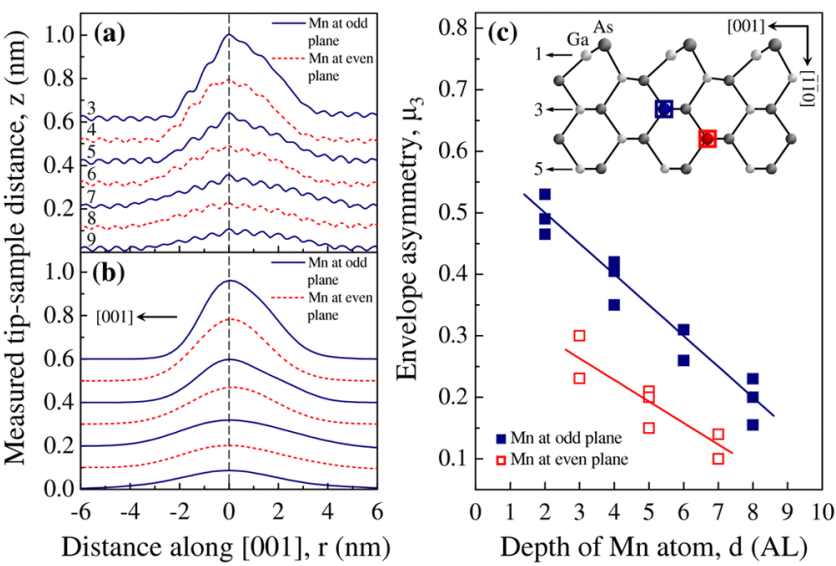

FIG. 2 (color online). (a) Topography along [001] and (b) FFT processed envelope line profiles along [001] of single Mn acceptors located between 2 and 8 AL below the GaAs(110) surface. The profiles are offset along the height axis for clarity. The (110) mirror plane showing the Mn symmetry is indicated by a dashed line through the contrast maximum. (c) The skewness of a number of envelope line profiles as a function of $\mathrm{Mn}$ depth below the GaAs(110) surface. The inset depicts the schematic side view of the GaAs(110) surface and the Mn atoms substituted on a Ga site in an odd or an even plane near the surface.

The asymmetry (skewness) of these envelope profiles is quantified by a standardized third moment, $\mu_{3}=$ $\left[\int_{-\infty}^{\infty}(r-\bar{r})^{3} Z(r) d r\right] / \sigma^{3}$, where $Z(r)$ corresponds to the envelope profile and $\sigma$ is the standard deviation of this profile. The third moment and thus the degree of asymmetry increase substantially for the $\mathrm{Mn}$ atoms closer to the GaAs(110) surface. Figure 2(c) shows the transition from the symmetric bulklike behavior to a strongly asymmetric shape for near surface $\mathrm{Mn}$ acceptors, with an interesting alternation between the Mn acceptors located in an odd or even layer.

Previously we have shown that multiband TB calculations are extremely useful to understand the spatial properties of acceptor states in III-V semiconductor materials $[3,14]$ and therefore we use again the approach developed in [15]. The results of the TB calculations for an unstrained bulk GaAs lattice, as presented in Fig. 3(b), show also an even or odd alternation which varies between $\mu_{3}=0.07$ and $\mu_{3}=0.08$ for even and odd layers, respectively. The measured $\mu_{3}$ for the calculated wave function is somewhat smaller than the experimentally obtained results which vary from 0.12 to 0.2 on average for deeply buried $\mathrm{Mn}$ acceptors. This demonstrates that, for depths greater than $8 \mathrm{AL}$ below the surface, the asymmetry of the bulk calculations is similar to that found experimentally. This observation is in contradiction with the prediction in Ref. [9]. The errors in modeling in [9] can likely be traced to the neglect of the $p$ - $d$ exchange interaction and the resulting error in the relative weight between the $T_{2}$-like and the $E$-like envelope functions [3]. 
In Fig. 3 we compare the topography images of the Mn acceptors located at different depths [Fig. 3(a)] with the corresponding TB calculations either in the absence [Fig. 3(b)] or in the presence [Fig. 3(c)] of a uniform strain. The TB calculations for an unstrained bulk lattice are sufficient to explain the spatial structure of Mn acceptors deep below the surface [3,5], but obviously fail for the acceptor states located near the surface. Recently we have shown that the shape of an acceptor wave function is highly sensitive to the local strain around an InAs quantum dot in GaAs [16]. The (110) surface of III-V semiconductors such as GaAs and InAs is relaxed and the shift in the lattice near the surface $[17,18]$ is described by a depth-dependent strain. To approximate the case of a relaxed GaAs surface within the TB model, the Ga lattice is shifted by $1.4 \mathrm{pm}$ ( $0.25 \%$ of the GaAs lattice constant) with respect to the As lattice along the [110] direction. The displacement between the two lattices is relatively small and corresponds to $10 \%$ of the actual vertical displacement between the $\mathrm{Ga}$ and As sites in the first subsurface layer below the GaAs(110) relaxed surface [17]. The applied shift in TB calculations induces a homogeneous internal strain in the $\mathrm{Ga}(\mathrm{Mn})$ As unit cell, whereas its volume is unchanged. In Refs. [5,19] surface related strain was also proposed to explain the breaking of the acceptor wave function symmetry near the InAs(110) surface, but without quantitative comparison.

The TB frames in Fig. 3(b) depict the (110) cross section of the Mn LDOS at different planes away from the Mn center in the GaAs crystal. The calculations in the absence

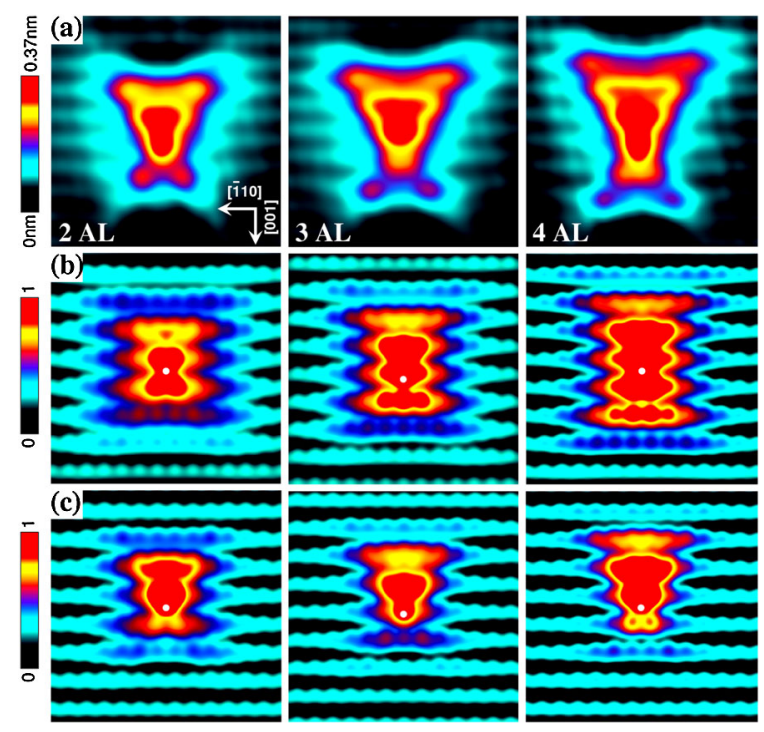

FIG. 3 (color online). (a) $\left(5 \times 5 \mathrm{~nm}^{2}\right) \mathrm{XSTM}$ topography images of single Mn acceptors located between 2 and 4 AL underneath the $\operatorname{GaAs}(110)$ surface. Calculated $\left(5 \times 5 \mathrm{~nm}^{2}\right)$ crosssectional TB view of Mn LDOS either in (b) the absence or in (c) the presence of a homogeneously strained $\mathrm{Ga}(\mathrm{Mn}) \mathrm{As}$ unit cell. The position of the $\mathrm{Mn}$ atom is indicated by a white spot. All the TB cross-sectional images retain a spin polarization oriented along [001] direction. of strain [Fig. 3(b)] reveal a mirror symmetry in the (110) plane and a relatively weak asymmetry in the [001] direction. As seen in Fig. 3(c) the applied shift of the Ga sublattice modifies the overall Mn LDOS and further breaks the symmetry of the Mn bound hole wave function along the [001] direction. Consistent with the topography images, the wing of the Mn wave function in the [001] direction is more pronounced than the wing in the opposite direction. The calculations mimicking the surface relaxation induced strain agree especially well for the Mn acceptors located at 2 and 3 AL below the surface, whereas it overestimates the symmetry breaking of the Mn acceptor at deeper layers. For example, in contrary to the experimental results as shown in Fig. 2(c), the absolute value of $\mu_{3}$ for the TB calculations for the strained bulk lattice is found to increase from 0.25 to 0.5 for the Mn wave functions at the second and eighth TB cross-sectional planes, respectively. This is no surprise because the surface relaxation induced strain disappears rapidly and inhomogeneously within a few atomic layers below the (110) surface of a real GaAs crystal.

The skewness $\left(\mu_{3}\right)$ [Fig. 2(c)] provides a relative scale for the value of the depth-dependent coupling of the Mn acceptors with the relaxed surface and gives a quantitative insight into the strain profile in the near surface layers. Unlike for deeply buried impurities, the bound hole localized near the vacuum interface interacts strongly with the surface relaxation induced strain and consequently its LDOS becomes highly asymmetric. We suggest that the alternating behavior of the asymmetry shown in Fig. 2(c) is related to the local bond reconfiguration at the topmost surface layers [17]. The nonuniform buckling of the surface and near surface atoms [see inset in Fig. 2(c)] gives rise to a strain undulation along the [001] direction at the surface with a periodicity equal to the separation of the $\mathrm{Ga}$ rows in the surface plane. Because the $\mathrm{Mn}$ acceptor is highly localized and its effective Bohr radius is comparable with the separation between the Ga rows, it might be affected by this periodic variation of the surface relaxation induced strain. When the Mn atom is located in an even (odd) plane, its projected position on the surface takes place between (under) the surface Ga rows, where we expect a local minimum (maximum) of the strain undulation along the surface. This can be understood by comparing the averaged actual displacements of the Ga atoms [17] in a vertical column beneath a $\mathrm{Ga}$ row and the averaged value in a vertical column between the surface Ga rows. Thus, the measurements suggest that the surface related strain, which breaks the Mn contrast symmetry, is bigger on average below a Ga row giving rise to a stronger breaking of wave function symmetry at these Mn positions. The behavior of odd or even alternation is further supported by TB calculations. The obtained $\left(\mu_{3}\right)$ parameters of the TB calculations for the unstrained bulk lattice show a much weaker (by a factor of $\sim 3$ ) odd or even alternation in depth than observed experimentally. This proves that there is no 


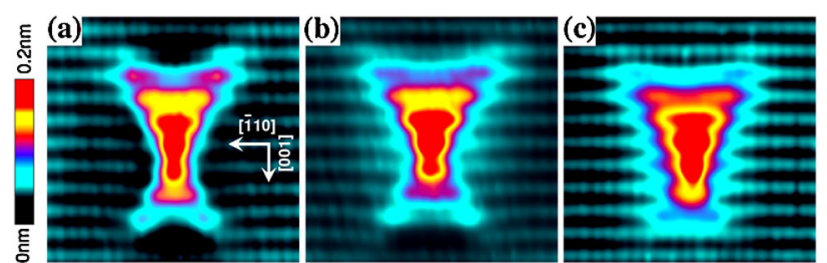

FIG. 4 (color online). $\left(6.5 \times 6.5 \mathrm{~nm}^{2}\right)$ topography images of single (a) Mn:GaAs, (b) $\mathrm{Cd}: \mathrm{GaP}$, and (c) $\mathrm{Zn}: \mathrm{GaP}$ acceptors located at $5 \mathrm{AL}$ below the (110) surface of the corresponding host crystal. The tunneling setpoint is the same for all the measurements $\left(V_{s}=+1.55 \mathrm{~V}, I_{t}=50 \mathrm{pA}\right)$.

major contribution from the $\left(T_{d}\right)$ symmetry of the bulk crystal.

We now extend this analysis to other acceptors and hosts. Observations of the asymmetry of true Coulombic impurities like $\mathrm{C}, \mathrm{Be}$, and $\mathrm{Zn}$ [with ground state binding energies $\left(E_{a}\right)$ around $30 \mathrm{meV}$ in InP and GaAs] show much more pronounced triangular shapes than $\mathrm{Mn}$. It is known that these shallow acceptors display a triangular contrast which extends laterally $\sim 5$ lattice constants on the cleavage surface $[2,4,6,10]$. Proposed explanations for the triangular appearance of the shallow acceptors include the electronic configuration of the outer shell $d$ electrons of different acceptor species [10], wave function mapping of the excited states retaining the zinc blende tetrahedral $\left(T_{d}\right)$ symmetry [4], and a resonant tunneling process involving evanescent states [6].

In Fig. 4 we compare the XSTM topography images of three acceptors with different binding energies [20] in GaAs and GaP. Each acceptor is located at 5 AL below the (110) surface of the respective host crystal. The acceptors with relatively large binding energy such as $\mathrm{Mn}: \mathrm{GaAs}$ $\left(E_{a}=113 \mathrm{meV}\right)$ [Fig. 4(a)] and Cd:GaP $\left(E_{a}=102 \mathrm{meV}\right)$ [Fig. 4(b)] display identical crosslike LDOS at the (110) surface. Cd showing this peculiar LDOS in GaP is very different from its previously reported triangular appearance in $\mathrm{GaAs}\left(E_{a}=35 \mathrm{meV}\right)$ [2]. This clearly indicates that the shape is related to the acceptor state and is not determined by the intrinsic property of the dopant. The response of the hole bound to $\mathrm{Zn}$, with a binding energy of $70 \mathrm{meV}$ in GaP [Fig. 4(c)], to the same strain field, is rather different from $\mathrm{Cd}$ state in GaP. The $\mathrm{Zn}$ LDOS is highly extended over the $\mathrm{GaP}(110)$ surface and shows a strong asymmetry in the [001] direction. For a fixed depth of an acceptor below the surface, the interaction of the bound hole with the surface relaxation induced strain changes with acceptor binding energy. The reduction of the LDOS symmetry with the binding energy is thus somewhat analogous with the depth-dependent change of the contrast symmetry. Acceptors with a smaller binding energy have a larger effective Bohr radius and thus their wave functions couple more strongly with the surface strain than the energetically deep acceptor states which are more spatially localized. This argument addresses why all the shallow acceptors such as $\mathrm{C}, \mathrm{Zn}$, and $\mathrm{Be}$ in GaAs appear as triangular features in STM measurements $[2,4,6,10]$.

XSTM has been used to investigate the LDOS of various acceptor states near the (110) semiconductor surface on the atomic scale. The experimentally observed depthdependent change of the Mn wave function symmetry was characterized quantitatively and interpreted successfully by TB calculations. We were able to show within the TB model that, without a detailed description of the reconstruction of the near surface layers and a vacuum halfsphere, the strain due to the relative displacement between the cation $(\mathrm{Ga})$ and the anion (As) sublattices is responsible for the experimentally observed reduction of the bulk acceptor wave function symmetry near the surface of III-V materials. With respect to the calculations presented in [9], which predicts the wrong asymmetry for acceptors deep below the surface, we argue that contrary to their explanation we image the acceptor wave function directly and that the surface relaxation related strain is the key component which explains the broken symmetry of both energetically shallow and near surface energetically deep impurities and the depth dependence of the symmetry breaking of deep acceptors in a unified approach.

The authors would like to thank A. M. Monakhov for valuable discussions. This work was supported by the Dutch Foundation for Fundamental Research on Matter (FOM), NanoNed (a technology program of the Dutch ministry of Economic Affairs via the foundation STW), VICI Grant No. 6631, and an ONR MURI.

[1] J. F. Zheng et al., Phys. Rev. Lett. 72, 1490 (1994).

[2] R. de Kort et al., Phys. Rev. B 63, 125336 (2001).

[3] A. M. Yakunin et al., Phys. Rev. Lett. 92, 216806 (2004).

[4] G. Mahieu et al., Phys. Rev. Lett. 94, 026407 (2005).

[5] F. Marczinowski et al., Phys. Rev. Lett. 99, 157202 (2007).

[6] S. Loth et al., Phys. Rev. Lett. 96, 066403 (2006).

[7] B. L. Sheu et al., Phys. Rev. Lett. 99, 227205 (2007).

[8] A.P. Wijnheijmer et al., Phys. Rev. Lett. 102, 166101 (2009).

[9] J.-M. Jancu et al., Phys. Rev. Lett. 101, 196801 (2008).

[10] Z. F. Zheng, M. B. Salmeron, and E. R. Weber, Appl. Phys. Lett. 64, 1836 (1994).

[11] J. K. Garleff et al., Phys. Rev. B 78, 075313 (2008).

[12] D. Kitchen et al., Nature (London) 442, 436 (2006).

[13] J.-M. Tang and M.E. Flatté, Phys. Rev. B 72, 161315 (2005).

[14] C. Çelebi et al., Phys. Rev. B 77, 075328 (2008).

[15] J.-M. Tang and M.E. Flatté, Phys. Rev. Lett. 92, 047201 (2004).

[16] A. M. Yakunin et al., Nature Mater. 6, 512 (2007).

[17] D. J. Chadi, Phys. Rev. B 19, 2074 (1979).

[18] B. Engels et al., Phys. Rev. B 58, 7799 (1998).

[19] S. Loth, M. Wenderoth, and R. G. Ulbrich, Phys. Rev. B 77, 115344 (2008).

[20] The acceptor binding energies are taken from $\mathrm{O}$. Madelung, Semiconductors-Basic Data (Springer, New York, 2004), 3rd ed. 